\title{
Automatisierte Methode zur Bestimmung von Chloridionen im Serum, Urin und Schweiß
}

\author{
Von H. Mahner, E. Henkel und A. Delbrück \\ Institut für Klinische Cbemie (Abteilung II) der Medizinischen Hocbscbule Hannover
}

(Eingegangen am 19. März 1973)

\begin{abstract}
Es wird über eine automatisierte Bestimmungsmethode für Chloridionen in geringster Konzentration berichtet. Die Nachweisgrenze liegt bei $7,8 \mu \mathrm{mol} / 1 \mathrm{Cl}$ im Testansatz. Die Bestimmung beruht auf der Bildung eines Eisen[III]-Chlorkomplexes in perchlorsaurem Milicu. Das Absorptionsmaximum liegt bei $348 \mathrm{~nm}$, die Messung kann aber ohnc weiteres bei $366 \mathrm{~nm}$ durchgeführt werden. Die automatisierte Methode ist zur Bestimmung von Chloridionen in Serum-, Harn- und Schweißproben geeignct. Sie ist als Mikro- bzw. als Ultramikromethode anzusehen und zeichnet sich durch eine gute Ubersichtlichkeit des Fließdiagramms aus. Der Variationskocffizient in der Serie für Serum beträgt $2,3 \%$, für Harn $2,1 \%$ und für Schweißproben $3,2 \%$.
\end{abstract}

\section{An automated method for the determination of cbloride ions in serum, urine and sueat}

$\Lambda \mathrm{n}$ automated method is described for the determination of very low concentrations of chloride ions. The detection limit is $7.8 \mu \mathrm{mol} / \mathrm{l}$ $\mathrm{Cl}$ in the sample. The determination is based on the formation of an iron[III]-chlorine complex in the presence of perchloric acid. The absorption maximum lies at $348 \mathrm{~nm}$, and satisfactory measurements can be made at $366 \mathrm{~nm}$. The automated method has been shown to be suitable for the determination of chloride in samples of serum, urine and sweat. It may be used as a micro, or ultramicro method, and the flow diagram is very easy to follow. The variation coefficient for serial assays is $2.3 \%$ for serum, $2.1 \%$ for urine, and $3.2 \%$ for sweat.

Der Bestimmung der Chloridkonzentration im biologischen Material ist seit langer Zeit große Aufmerksamkeit geschenkt worden. Als essentieller Bestandteil menschlicher Körperflüssigkeiten des intra- und extrazellulären Raumes besitzt das Chlorid als biologischer Parameter sowohl für die klinische Diagnose als auch für pathogenetische Fragestellungen eine große Bedeutung. Es wurden bisher eine Reihe von Verfahren zur Bestimmung der Chloridkonzentration im Blutserum und anderen Körperflüssigkeiten entwickelt, die in unterschiedlichem Maße Verbreitung gefunden haben.

Eine ausführliche Diskussion der gebräuchlichsten Chlorid-Bestimmungs-Methoden gibt Cotrove (1).

Die heute gebtäuchlichsten Methoden sind:

1. Coulometrische Titration nach Cotrove und Mitarbeitern.

2. Merkurimetrische Titration nach Schales und SCHALES.

3. Kolorimetrische automatisierte Chloridbestimmung mit Quecksilberthiocyanat nach SkEGGS und HockSTRASSER.

Die unter 1 und 3 genannten Verfahren sind zur Bestimmung von Chloridionen in niedriger Konzentration zu unempfindlich. Das an zweiter Stelle genannte Verfahren ist als Ultramikromethode für sehr niedrige Chloridkonzentrationen geeignet, ist aber wegen des hohen Arbeitsaufwandes für die einzelne Probe in seinen Anwendungsmöglichkeiten begrenzt. Es ergab sich die Notwendigkeit für größere tierexperimentelle Versuchsreihen bzw. für die Untersuchung größerer Kollektive zur Bestimmung von Chlorid in Proben niedriger Chloridkonzentration z. B. Schweiß, nach anderen Mög- lichkeiten der Chloridbestimmung zu suchen. Von WEST und ColL wurde 1956 ein spektrophotometrisches Verfahren (2) angegeben, welches auf der Bildung eines Chlorkomplexes mit dreiwertigem Eisen beruht. Dieses Verfahren wurde von KUPKE $(3,4)$ zur Bestimmung von Chlorid im tierischen Gewebe und Serum modifiziert.

Ziel der vorliegenden Untersuchung war es, zu prüfen, wie weit dieses Verfahren für eine automatische Bestimmung in einem kontinuierlichen Analysensystem geeignet ist, insbesondere sollte die Anwendbarkeit auf niedrige Chloridkonzentrationen untersucht werden.

\section{Material und Methoden}

Geräte

1. Photometer Eppendorf M 1101

2. Schreiber: Lin-log-converter, Typ UR 400 der Fa. Vitatron

3. Proportionierungspumpe: Auto-Analyzer

Proportioning Pump der $\mathrm{Fa}$. Technicon

4. Probennehmer: Fa. Technicon

5. Küvette: Durchflußküvette Eppendorf 4053

6. Schlauchmaterial: Technicon Pumpenschläuche, Teflon Verbindungsschlauch, Einzelheiten siehe Fließbild

Chemikalien

1. Eisen[III]-perchlorat purum (Fluka A. G., Buchs S. G. Nr. 44960)

2. Perchlorsäure p. a. etwa $700 \mathrm{~g} / \mathrm{kg}(11,6 \mathrm{~mol} / 1)$ (Merck Art 519; Verunreinigung mit $\mathrm{Cl}-$-Ionen maximal $0,0003 \%$ )

3. Normal Clinical Chemistry Control Serum (Hyland)

4. Abnormal Clinical Chemistry Control Serum (Hyland)

5. $\mathrm{NaCl}$ Suprapur (Merck Nr. 6406)

6. Serumalbumin vom Rind, trocken reinst, ORHD 20/21 (BehringWerke)

7. Brij 35 (Polyoxyäthylenlauryläther) (Mcrck 1962)

Herstelling der Reagenzien

1. Eisenreagenz: $4 \mathrm{~g} \mathrm{Fe}\left(\mathrm{ClO}_{4}\right)_{3}$ in etwa $5 \mathrm{ml}$ bidest. Wasser lösen und mit $700 \mathrm{~g} / \mathrm{kg} \mathrm{HClO}_{4}$ auf $500 \mathrm{ml}$ auffüllen. 
2. Vcrdünnungslösung: $100 \mathrm{~g} / \mathrm{kg} \mathrm{HClO}_{4}(1,6 \mathrm{~mol} / \mathrm{l})(84 \mathrm{ml} 700 \mathrm{~g} / \mathrm{l}$ $\mathrm{HClO}_{4}$ mit bidest. Wasser auf $500 \mathrm{ml}$ auffüllen und $0,5 \mathrm{ml}$ Brij 35).

In dunkler Flasche im Kühlschrank aufbewahren.

Herstellung der Eich- und Testlösungen

$1.2 \mathrm{~mol} / 1 \mathrm{NaCl}$ - Stammlösung: $11,688 \mathrm{~g} \mathrm{NaCl}$ als Urtitersubstanz (im Vakuum bei $105^{\circ} \mathrm{C}$ bis zur Gewichtskonstanz getrocknet) mit bidest. Wasser auf $100 \mathrm{ml}$ auffüllen.

2. Wäßrige Eichlösungen: $20 \mathrm{mmol} / 1 \mathrm{NaCl}-180 \mathrm{mmol} / 1 \mathrm{NaCl}$.

3. Rinderalbuminlösung: $100 \mathrm{~g} / 1$

4. Eichlösungen mit einem Albumingehalt von $70 \mathrm{~g} / \mathrm{l}$ für die Messung von Serumproben : $70-130 \mathrm{mmol} / 1 \mathrm{NaCl}$

5. Normal Clinical Chemistry Control Serum (Hyland) Sollwert $108 \mathrm{mmol} / 1 \mathrm{Cl}^{-}$

6. Abnormal Clinical Chemistry Control Serum (Hyland) Sollwert $126 \mathrm{mmol} / \mathrm{l} \mathrm{Cl}^{-}$

\section{Untersuchungsmateria}

Die Serum- und Urinproben entstammen dem Untersuchungsgut der Abteilung II des Institutes für Klinische Chemie, Zentrallabor des Krankenhauses Oststadt, der Medizinischen Hochschule Hannover. Die Schweißproben wurden von klinisch-chemischen Laboratorien der Universitäts-Kinderklinik Hamburg-Eppendorf bzw. des Allgemeinen Krankenhauses Heidberg (Hamburg) freundlicherweise zur Verfügung gestellt ${ }^{1}$ ).

\section{Vorgehen}

Serum- und Urinproben

Entsprechend dem in Abbildung 1 dargestellten Fließbild wurde die Chloridbestimmung an nativen Serum- und Urinproben im kontinuierlichen Analysensystem wie folgt durchgeführt. Es werden - bei einer Probenfrequenz von 30 Proben pro Stunde und eincm Verhältnis von Probe zu Spülflüssigkeit 1:2-0,10 ml Untersuchungsmaterial für eine Bestimmung angesaugt. Im Verlaufe der Analyse wird die Probe mit bidest. Wasser im Verhältnis $1: 25$ unter Luftsegmentierung vorverdünnt. Ein Aliquot dieser Serumverdünnung wird in ein luftsegmentiertes Reagenzgemisch eingeleitet. Bei Raumtemperatur exfolgt eine Inkubation des Gemisches in einer Verzögerungs-Spirale für etwa 10 Minuten. Anschließend wird im Photometer mit Durchflußküvette bei $366 \mathrm{~nm}$ die Extinktion fortlaufend nach Linearisierung mit einem Schreiber registriert. Die Auswertung der den Serumproben entsprechenden aufgezeichneten Peaks erfolgt anhand einer eiweißhaltigen Eichkurve, die Auswertung der Urinproben mit einer wäßrigen Eichkurve.

\section{Scloveißproben}

Die Gewinnung der Schweißproben erfolgt mittels der PilocarpinIontophorese. Durch die Einwirkung von Gleichstrom dringt Pilocarpin in die Haut ein (Iontophorese). Das Parasympathicomimeticum stimuliert die Schweißdrüsensekretion. Der Schweiß wird von saugfähigem Filterpapier oder Mull aufgenommen. Durch Wägung vor und nach Durchführung der Iontophoreșe wird die Menge des abgesonderten Schweißes ermittelt. Anschließend wird die Schweißprobe aus dem Filter gewaschen. Bei diesem Waschvorgang wird eine Verdünnung von 1:25 erreicht.

Für die Bestimmung der Chloridkonzentration im Schweiß wird entsprechend der Abbildung 1 und dem unter Serum- bzw. Urin beschriebenen Verfahren vorgegangen. Allerdings entfällt im Analysensystem entsprechend der bei der Schweißgewinnung vorgenommenen Verdünnung der Probe die automatische Vorverdünnung. Die Auswertung exfolgt über eine besondere Eichkurve, die im Verhältnis 1:25 vorverdünnt angesetzt wird.

In dem geschilderten Verfahren werden die Analysen in einem Meßbcreich von 5-140 $\mu \mathrm{mol} / 1$ Chloridkonzentration durchgeführt. In diesem Bereich ist das LAMBERT-BEER'sche Gesetz erfüllt, so daß die Eichkurve linear verläuft.

1) Wir danken Frau Dr. RYBak und Herrn Dr. Fụ̈r für die Úberlassung des Untersuchungsmaterials.

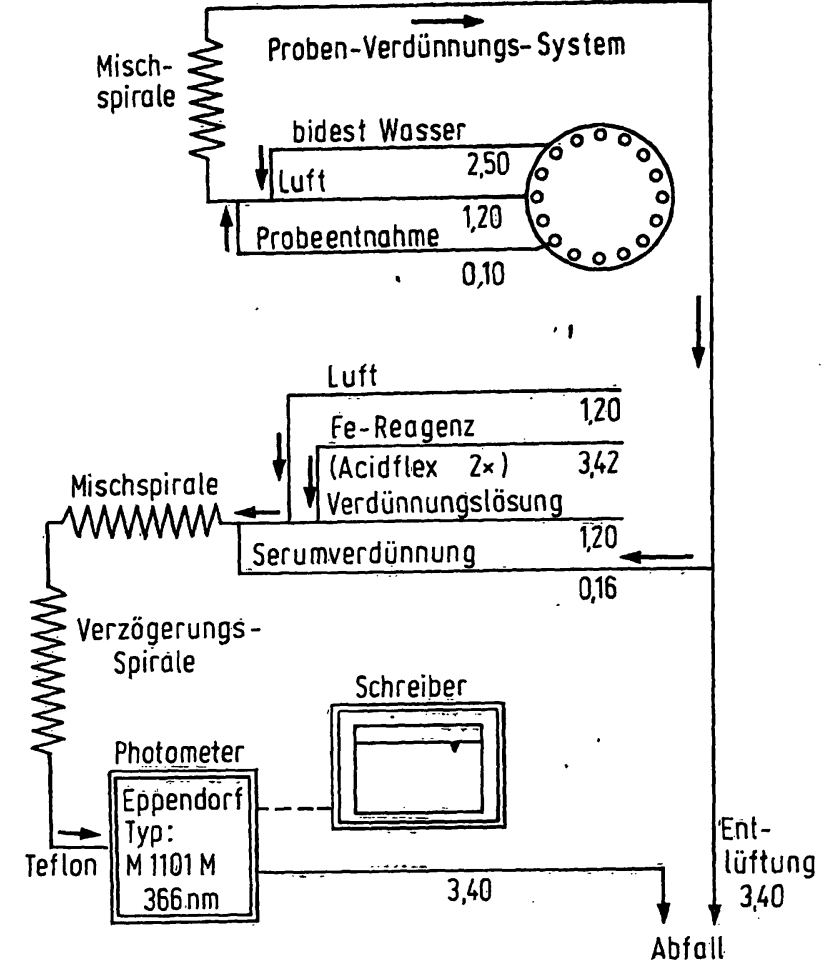

Abb. 1

Fließ̣diagramm żur automatischen Chlorid-Bestimmung im Serum, Urin und Schweiß. Die Zahlenangaben an den Pumpenschläuchen bezeichnen den Proben- bzw. Reagenziendurchflu $\beta$ in $\mathrm{ml} / \mathrm{min}$

\section{Ërgebnisse}

Die Ưberprüfung der Präzision in der Serie wurde durch Messungen von Serumproben durchgeführt. Die Ergebnisse sind in Tabelle 1 dargestellt. Die Richtigkeit der Meßergebnisse wurde durch Vergleich mit den Werten käuflicher Testseren (Hyland) untersucht (Tab. 2). Als Vergleich dazu dienten die Werte der gleichen Testseren aus der Qualitätskontrolle im Routinelabor, die mit dem Clor-o-counter ermittelt wurden. In Wiederfindungsversuchen mit Zumischung von proteinhaltigen Lösungen hoher und niedriger Chloridkonzentrationen. zu verschiedenen Serumproben im Verhältnis $1: 2$ wurden die Daten der Tabelle 3 ermittelt. Die Variationskoeffizienten für die Präzision in der Serie betrugen 2,29\% bzw. 1,18\%. Die mittlere Abweichung bei der Richtigkeitsprüfung lag bèi $+0,8 \%$ bzw. $+0,3 \%$. Die Wiederfindungsrate betrug $99 \%$.

Die entsprechenden Daten für die Urinproben gehen aus Tabelle 1 hervor. Die Präzision in der Serie, ausgedrückt als Variationskoeffizient, ergab $2,1 \%$ bzw. $1,37 \%$ bzw.

Tab. 1

Präzisionskontrolle in der Serie an verschiedenen Untersuchungsmaterialien

$\mathrm{VK}_{\mathrm{S}}=$ Variationskoeffizient in der Serie

\begin{tabular}{lrccc}
\hline Probe & $\mathrm{n}$ & $\overline{\mathrm{x}}$ & $\mathrm{s}$ & $\mathrm{VKs}$ \\
\hline Serum & 21 & $106,4 \mathrm{mmol} / 1$ & 2,44 & $2,29 \%$ \\
& 12 & $130,2 \mathrm{mmol} / 1$ & 1,54 & $1,18 \%$ \\
Harn & 36 & $84,3 \mathrm{mmol} / 1$ & 1,79 & $2,1 \%$ \\
& 9 & $102,7 \mathrm{mmol} / 1$ & 1,41 & $1,37 \%$ \\
& 14 & $150,7 \mathrm{mmol} / 1$ & 1,97 & $1,31 \%$ \\
Schweiß & 20 & $46,53 \mathrm{mmol} / 1$ & 1,51 & $3,23 \%$
\end{tabular}


Tab. 2

Rlchtigkeitskontrolle der automatischen Chloridbestimmung an Kontroliseren im normalen und abnormalen Bereich (Hyland) sowie an einem wäßrigen Standard zur Kontrolle des Verfalırens bei Urinproben, in Vergleich zu Wertell aus der Qualitătskontrolle im Routinelabor der gleichen Kontrollseren

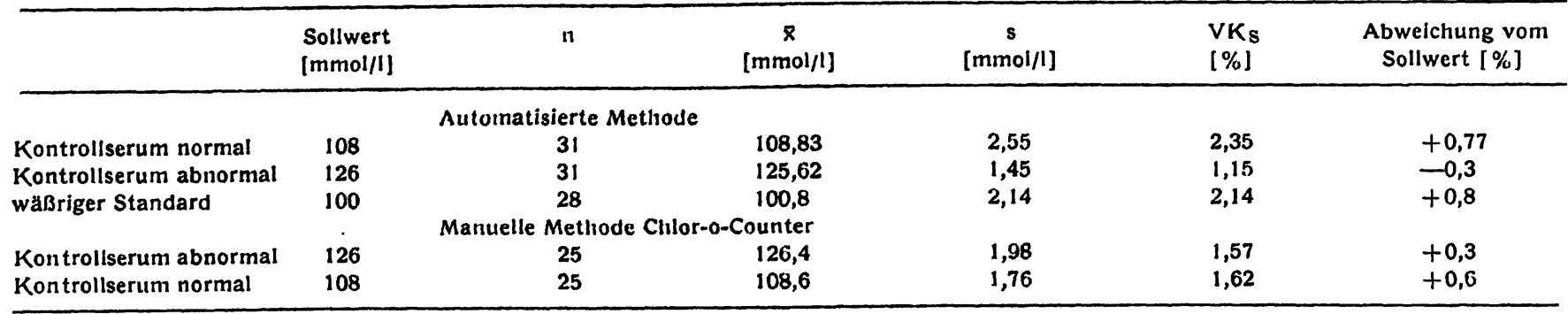

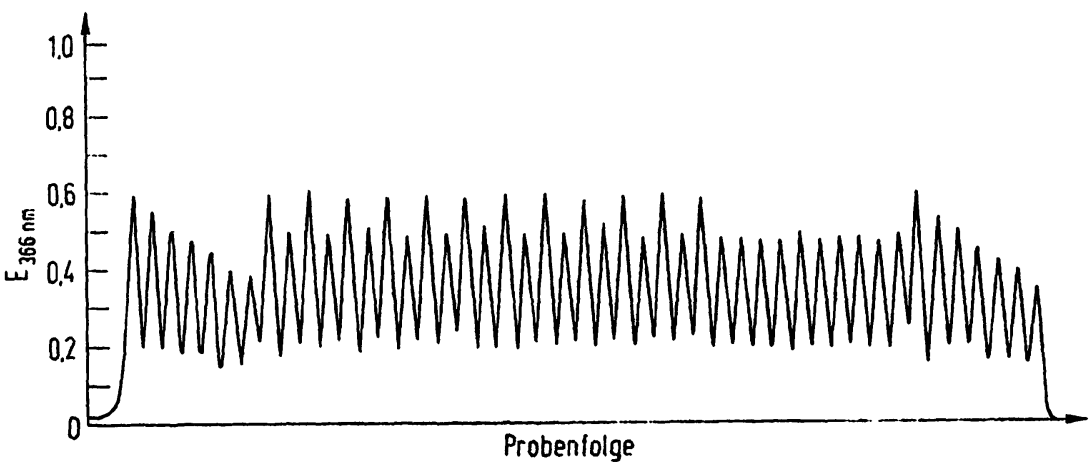

Abb. 2

Öberprüfung der Präzision in der Serie für Serumproben

1,31\%. Die Abweichung von der Richtigkeit war kleiner als $0,1 \%$ und die Wiederfindungsraten lagen im Mittel bei $103 \%$.

Die Überprüfung der Präzision in der Serie für das Verfahren zur Messung an Schweißproben wurde mit wäßrigen NaCl-Lösungen durchgeführt. Der Variationskoeffizient bei dieser Serie lag bei 3,23\% (Tab. 1). Bei einem Mittelwert von 19,3 mmol/l Chlorid lagen die Werte für die Schweiß-Konzentrationen - die nach dem oben geschilderten Verfahren an einem Kollektiv von 43 Personen ermittelt wurdeu - bei allen untersuchten Probanden im Normbereich. Der niedrigste Wert betrug 5,1 mmol/l, der höchste, dem Normalbereich zugeordnete Meßwert betrug 35,6 mmol/1. BENDER und STEPHAN (5) geben als Mittelwert eine Chloridkonzentration von $20 \mathrm{mmol} / \mathrm{l}$ bei einem Bereich zwischen 10 und $40 \mathrm{mmol} / \mathrm{l}$ an. Die nach Schales bzw. nach LaNG an anderem Ort vorgenommenen Parallelbestimmungen ergaben einen Mittelwert von $18,5 \mathrm{mmol} / \mathrm{l}$ mit einem Bereich von 6,0 bis $50,0 \mathrm{mmol} / \mathrm{l}$. Unsere Messung bestätigt bei einem erhöhten $(55,6 \mathrm{mmol} / \mathrm{l})$ Meßwert das Ergebnis der Parallelbestimmung. In diesem Falle liegt der Verdacht einer Mucoviscidose vor, aus äußeren

Tab. 3

Wiederfindung bei Zumischung von proteinhaltigen Eichlösungen $\mathrm{zL}$ Serumproben im Verhältnis 1:2 für 2 Konzentrationsbereiche bzw. wäßriger Eichlösung zu Urinproben (1:2)

\begin{tabular}{lrrrr} 
& n & \multicolumn{2}{c}{$\begin{array}{c}\text { Zugabe } \\
{[\mathrm{mmol} / 1]}\end{array}$} & $\begin{array}{c}\text { Wiederfindung } \\
{[\%]}\end{array}$ \\
\hline \multirow{2}{*}{ Serum } & 32 & 70 & $1: 2$ & $99,0 \pm 2,5$ \\
\multirow{3}{*}{ Urin } & 32 & 130 & $1: 2$ & $99,2 \pm 2,2$ \\
& 32 & 70 & $1: 2$ & $103,0 \pm 2,3$ \\
& 32 & 130 & $1: 2$ & $104,6 \pm 1,3$ \\
\hline
\end{tabular}

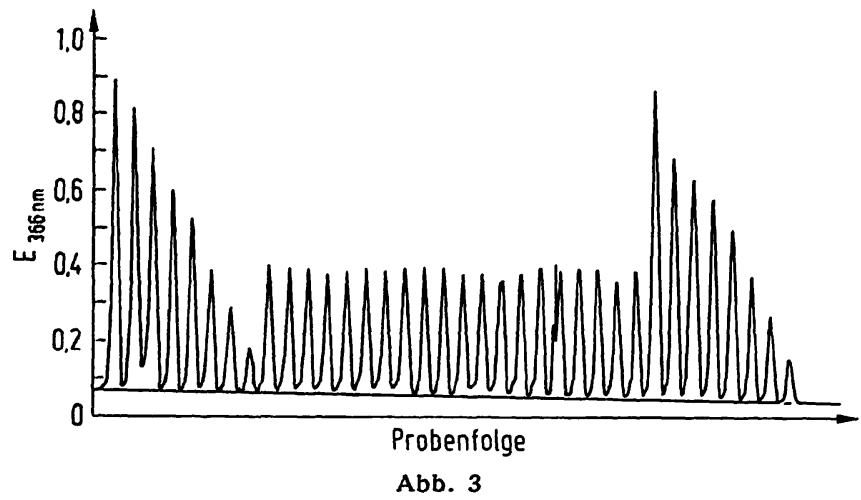

Überprüfung der Präzision in der Serie für Schweißproben

Gründen konnte bei diesem Probanden die Diagnostik nicht zum Abschluß gebracht werden.

Die von KUPKE gemachte Beobachtung eines Extinktionsanstieges bei Verwendung eiweißhaltiger Lösungen

Tab. 4

Einfluß des Proteingehaltes von Untersuchungsproben auf die Blindextinktionen der einzelnen Reaktionspartner. Es wurden jeweils $0,05 \mathrm{ml}$ bidest. Wasser bzw. $0,05 \mathrm{ml} 100 \mathrm{~g} / \mathrm{l}$ Albumin-Lösung zu $1 \mathrm{ml}$ Reaktionslösung zugesetzt

\begin{tabular}{|c|c|c|c|c|}
\hline \multicolumn{2}{|l|}{ Reaktionslösung } & \multirow{2}{*}{$\begin{array}{c}\begin{array}{c}\text { bidest. } \\
\text { Wasser } \\
\text { Ext. } 1\end{array} \\
\text { z } 0,131\end{array}$} & \multirow{2}{*}{$\begin{array}{c}\begin{array}{c}100 \mathrm{~g} / 1 \\
\text { Albumin } \\
\text { Ext. } 2\end{array} \\
0,180\end{array}$} & \multirow{2}{*}{$\begin{array}{c}\begin{array}{l}\text { Differenz } \\
\left(E_{2}-E_{2}\right)\end{array} \\
+0,049\end{array}$} \\
\hline $\begin{array}{l}\text { Gebrauchs- } \\
\text { lösung }\end{array}$ & $\begin{array}{l}75 \text { Teile Fe-Reagenz } \\
+20 \text { Teile Ver- } \\
\text { dünnungslösung }\end{array}$ & & & \\
\hline $\begin{array}{l}\mathrm{HClO}, \\
\text { Verdünnungs- } \\
\text { lösung }\end{array}$ & $\begin{array}{l}\text { etwa } 700 \mathrm{~g} / \mathrm{kg} \\
100 \mathrm{~g} / \mathrm{kg} \mathrm{HClO}\end{array}$ & $\begin{array}{l}0,008 \\
0\end{array}$ & $\begin{array}{l}0,120 \\
0,250\end{array}$ & $\begin{array}{l}+0,112 \\
+0,250\end{array}$ \\
\hline Fe-Reagenz & $\begin{array}{l}4 \mathrm{~g} \mathrm{Fe}\left(\mathrm{ClO}_{4}\right)_{3} \text { in } \\
5 \mathrm{ml} \text { bidest. Wasser } \\
\text { gelöst }+500 \mathrm{ml} \\
70 \mathrm{~g} / \mathrm{kg} \mathrm{HClO}\end{array}$ & 0,265 & 0,386 & $+0,121$ \\
\hline Bidest. Wasser & & 0 & 0,025 & $+0,025$ \\
\hline
\end{tabular}


konnte in der vorliegenden Studie bestätigt werden. Der Zusatz chloridfreien Albumins löste das gleiche Phänomen aus. In der verwendeten Lösung wurde durch coulometrische Titration bzw. Silbernitrat die Anwesenheit von Chlorionen ausgeschlossen. Das Auftreten von sichtbaren Trübungen wurde nicht beobachtet. Sowohl bei Albuminzusatz zum vollständigen Reagenz wie bei der Zugabe von Albumin zu den einzelnen Komponenten des Reaktionsgemisches kommt es zu einer mehr oder weniger starken Ausprägung des beobachteten Effektes. Die Ergebnisse sind in Tabelle 4 dargestellt.

\section{Diskussion}

Das von West und Coll (2) 1956 angegebene DirektVerfahren zur spektrophotometrischen Bestimmung kleiner Chloridmengen beruht auf der Bildung eines Chlorkomplexes mit dreiwertigem Eisen. Dieser Komplex besitzt ein Absorptionsmaximum bei $348 \mathrm{~nm}$.

1969 wurde von KUPKE eine Modifikation dieses Chloridbestimmungsverfahrens für tierische Gewebe, für Blutserum und andere Körperfüssigkeiten angegeben.

Basierend auf dieser Modifikation wurde unter Verwendung der vorgeschlagenen Reagenzien die Methode auf die Anwendungsmöglichkeit in einem kontinuierlichen Fließsystem untersucht (6), neben der Chloridbestimmung im Serum und Urin wurde besonders auf die Messung von Chloridkonzentrationen im Schweiß eingegangen, da diese für die Diagnostik der Mucoviscidose von Bedeutung ist.

WEST und CoLL gingen in ihrer Untersuchung bereits davon aus, daß Eisen[III]-Ionen in Lösung nicht, wie lange angenommen wurde, gelb gefärbt sind, sondern farblos sind. Die beobachtete gelbe Farbe rühtt von Chlor bzw. Hydroxokomplexen her. Sie untersuchten daher die Verwendbarkeit von chloridfreiem Eisen für die kolorimetrische Bestimmung von Chloridionen und fanden, daß das Maximum der Absorption bei $348 \mathrm{~nm}$ in erster Linie von der Konzentration der Säure im Bestimmungsansatz abhängig sei. Unter den gebräuchlichen starken Säuren erwies sich die Perchlorsäure als am geeignetsten, da der Eisenperchloratkomplex wenig Energie in dem für die Chloridbestimmung geeigneten Spektralbereich absorbiert und unter den Versuchsbedingungen aus Perchlorsäure freigesetzte Chloridionen nicht nachgewiesen werden konnten. Eine Interferenz von Jodid- bzw. Bromidionen bei dem angegebenen Bestimmungsverfahren liegt nicht vor, dagegen wirken diese Halogene bei den herkömmlichen Chloridbestimmungsverfahren in beträchtlichem Ausmaß als Störfaktoren.

Alle anderen in biologischen Systemen anwesenden Ionen zeigen keinerlei Interferenzen in den dort auftretenden Konzentrationen. Das gilt auch für die Konzentrationen des Serumeisens bzw. Hämoglobins (4), da im Testansatz mit einem hohen Eisenüberschuß gearbeitet werden muß, stellt die geringe Eisenkonzentration im Serum keinen Störfaktor dar. Sulfationen stellen bis zu einer Konzentration, die etwa der Hälfte der Chloridkonzentration im Serum entspricht, keinen Störfaktor dar. Diese Konzentrationsverhältnisse kommen im biologischen Material nicht vor. Die Sulfatkonzentration im Serum beträgt $0,5 \mathrm{mmol} / \mathrm{l}$. Die günstigsten Reaktionsbedingungen wurden durch Einsatz konzentrietter Perchlorsäure erzielt. Mit der Verwendung von konzentrierter Perchlorsäure sind allerdings eine Reihe von praktischen Problemen verbunden. So kommt es aufgrund der starken Hygroskopie der Perchlorsäure zu Drifterscheinungen in der Basislinie durch Aufnahme von Wasser aus der umgebenden Luft. Ebenfalls werden die mechanischen Eigenschaften der verwendeten Pumpenschläuche durch konzentrierte Perchlorsäure erheblich beeinträchtigt. Bei Verwendung einer verdünnten Perchlorsäure findet sich zwar nicht die gleich hohe Empfindlichkeit wie bei konzentrierter Perchlorsäure, jedoch vermeidet man die ins Gewicht fallenden Driften und stärkere Beeinträchtigungen der Pumpenschläuche. Es erwies sich als ausreichend, das Schlauchmaterial wöchentlich zu wechseln. Eine auftretende Drift war jeweils eir Zeichen für eine nicht mehr tolerierbare Alterung der Schläuche.

Trotz Verwendung verdünnter Perchlorșäure ist das Reagenz möglichst unter Verschluß vor Luftfeuchtigkeit und gegen Licht geschützt aufzubewahren. Besondere Beachtung verdient auch die Proteinkonzentration der Probe beim Eintritt in das Reagenz. Aufgrund von Vorversuchen wurde als günstigstes Verdünnungsverhältnis für Serum im Vorverdünnungsanteil der Analyse ein Verhältnis 1:25 gefunden. Bei dieser Vorverdünnung bleiben bei Eintritt der eiweißhaltigen Probe in das perchlorsäurehaltige Reagenz die Proteine in Lösung und verursachen keine störenden Trübungseffekte.

Die unspezifische Extinktionserhöhung durch Proteine, bedingt durch die Absorption bei $366 \mathrm{~nm}$, konnte durch Verwendung einer eiweißhaltigen Eichlösung ausgeglichen werden. Durch diese Maßnahme kann auf die Enteiweißung der Probe verzichtet werden, die bei einer rein wäßrigen Eichlösung unbedingt erforderlich ist. Das Ausschalten eines Deproteinisierungsschrittes ist sowohl in der Handmethode wie auch bei einem automatischen Verfahren von großem Vorteil, zumal bei dem letzteren der sehr störanfällige Dialyseschritt zur Enteiweißung ausgeschaltet ist.

Die Absorption gelöster Proteine im sauren Milieu bei $366 \mathrm{~nm}$ spielt u. a. auch bei der Blutglucosebestimmung eine Rolle und wurde in einer ausführlichen Studie untersucht (HENkEL, E. in Vorbereitung).

Die sehr hohe Empfindlichkeit weist diese quantitative Chloridnachweismethode als Ultramikromethode aus und erlaubt das Arbeiten mit extrem niedrigem Probeneinsatz. Durch die große Variationsmöglichkeit in der Vorverdünnung besitzt diese Methode eine große Flexibilität in Bezug auf die im biologischen Untersuchungsmaterial vorkommenden Chloridkonzentrationen. Durch Variation der automatischen Vorverdünnung können alle Konzentrationsbereiche erfaßt werden. Serum- und Urinproben lassen sich mit dem gleichen Vorverdün-. 
nungssystem analysieren. Zur Bestimmung der Schweißproben wird das Vorverdünnungssystem nicht benötigt, da bei der Elution der Proben ein Verdünnungsverhältnis von 1:25 erreicht wird. Gerade die Möglichkeit des geringen Probenbedarfs legt es nahe, diese Chloridbestimmungsmethode auch in Vielfachanalysatoren anzuwenden, da eine Reduzierung der erforderlichen Serummengen für die einzelnen in Vielfachanalysatoren kombinierten Verfahren von wesentlicher Bedeutung ist.

Verzichtet man auf die automatische Probenverdünnung und verwendet einen Dilutor, so reichen $10 \mu \mathrm{l}$ Serum bzw. Urin als Probenmenge zur Bestimmung aus. Das Verdünnungsverhältnis in der Küvette beträgt 1:50 und beinhaltet damit noch einigen Spielraum, die Relation Probe zu Reagenz abzuändern, um mit noch weniger Probe auszukommen oder auch noch niedrigere Kon- zentrationen zu erfassen. Die Nachweisgrenze im Fließdiagramm für Schweißproben liegt bei $7,8 \mu \mathrm{mol} / 1$ Testansatz. Bis zu dieser Konzentration ist eine eindeutige und exakte Ablesung der Peaks unabhängig von eventuellen Störsignalen (noise) möglich. Das Signal auf dem Schreiber beträgt hierbei $4 \%$ der gesamten Skalenbreite. Die im Flicßbild deutlich werdende Übersichtlichkeit des Fließ-Systems, seine geringe Störanfälligkeit und der geringe Arbeitsaufwand, verbunden mit dem großen Konzentrationsbereich, der mit dieser Methode erfaßt werden kann, öffnet diesem Analysenverfahren ein breites Anwendungsfeld, wobei insbesondere die Automatisierbarkeit von großem Vorteil für die Analytik großer Untersuchungsreihen ist. Gerade im Hinblick auf die Vorteile bei ciner Automatisierung zeigt sich das hier beschriebene Verfahren den hergebrachten Chloridbestimmungsmethoden überlegen.

\section{Literatur}

1. Cotrove, E. (1964), Methods Biochem. Anal. 12, 277-391. 2. West, Pri. \& Coll, H. (1956), Anal. Chem. 28, 1834-1838. 3. Kupke, I. (1969), diesc Z. 7, 599-600. - 4. KUPKE, I. \& SAURR, D. (1970), Z. Anal. Chem. 250, 101-104. - 5. Bendek, S. W. \& Stephan, U. (1970), Mucoviscidosc, Cystische Fibrose, S. 43. Dcutsche Gescllschaft zur Bekämpfung der Mucoviscidosc e. V.
Erlangen. - 6. Hrinket, E., MAHNer, H. \& KupKe, I. (1970), Zur Chloridbestimmung an Proben niedriger Kon\%entration im Auto-Analyzer-System (Vortrag Technicon Symposium Bad Homburg). - 7. Insorr, F. A. (1965), Standard Methods Clin. Chem. 5, 101-111.
Anschrift der Verfasser: Institut für Klinische Chemie (Abteilung II) der Medizinischen Hochschule Hannover Zentrallabor im Krankenhaus Oststadt 3000 Hannover, Podbielskistraßc 380 